\title{
Numerical Investigation on Feedback Insensitivity in Semiconductor Nanolasers
}

Fan, Yuanlong; Hong, Yanhua; Li, Pui

\section{IEEE Journal of Selected Topics in Quantum Electronics}

DOI:

10.1109/JSTQE.2019.2899765

Published: 01/11/2019

Peer reviewed version

Cyswllt i'r cyhoeddiad / Link to publication

Dyfyniad o'r fersiwn a gyhoeddwyd / Citation for published version (APA):

Fan, Y., Hong, Y., \& Li, P. (2019). Numerical Investigation on Feedback Insensitivity in Semiconductor Nanolasers. IEEE Journal of Selected Topics in Quantum Electronics, 25(6). https://doi.org/10.1109/JSTQE.2019.2899765

\footnotetext{
Hawliau Cyffredinol / General rights

Copyright and moral rights for the publications made accessible in the public portal are retained by the authors and/or other copyright owners and it is a condition of accessing publications that users recognise and abide by the legal requirements associated with these rights.

- Users may download and print one copy of any publication from the public portal for the purpose of private study or research.

- You may not further distribute the material or use it for any profit-making activity or commercial gain

- You may freely distribute the URL identifying the publication in the public portal?
}

Take down policy

If you believe that this document breaches copyright please contact us providing details, and we will remove access to the work immediately and investigate your claim. 


\title{
Numerical Investigation on Feedback Insensitivity in Semiconductor Nanolasers
}

\author{
Yuanlong Fan, Yanhua Hong, Member, IEEE and Pu Li
}

\begin{abstract}
This paper presents numerical investigations into the effect of feedback phase on the stability of semiconductor nanolasers (SNLs) in presence of the external optical feedback (EOF). For this purpose, numerical solutions are obtained from rate equations where the effects of Purcell cavity-enhanced spontaneous emission factor $F$ and an enhanced spontaneous emission coupling factor $\beta$ are included. In this way, a phase-insensitive stable SNL is identified when the feedback coupling fraction is below a critical threshold, $\eta_{\text {c. Furthermore, }}$ the relationship between $\eta_{c}$ and two other important system parameters, namely the injection current $\left(I_{\mathrm{dc}}\right)$ and the initial external cavity length $\left(L_{0}\right)$, is studied. The results show that $\eta_{\mathrm{c}}$ has a bi-exponential relationship with either $I_{\mathrm{dc}}$ or $L_{0}$. Moreover, the influence of $F$ on the $\eta_{c}$ is evaluated and it is found that $\eta_{c}$ increases with the increase of $F$. The results presented in this paper provide practical guidelines for the design of phase-insensitive stable SNLs which are useful for densely integrated photonic circuits based applications such as optical communications and sensing.
\end{abstract}

Index Terms-Optical feedback, photonic integrated circuits, semiconductor nanolasers.

\section{INTRODUCTION}

$\mathrm{I}^{\mathrm{N}}$ $\mathrm{N}$ the past two decades, considerable progress in miniaturing lasers has been achieved along with the development of integrated photonic circuits and system-on-a-chip technologies where light sources are required. As part of that effort, considerable attention has been given to the design of semiconductor nanolasers (SNLs) [1] which offer ultra-small footprints (typically being smaller than the free-space laser wavelength in all three dimensions) and compatibility with existing photonic integrated circuits (PICs) platforms. In the meanwhile, interesting physical aspects of SNLs have been recognized including the potential for demonstrating enhanced coupling efficiency of spontaneous emission into the lasing cavity mode via the Purcell effect [2]. Such enhanced

This work is supported by United Kingdom Engineering and Physical Sciences Research Council (U.K. EPSRC Grant No. EP/P006027/1) and National Natural Science Foundation of China (NNSFC Grant No.61505137 and 61775158).

Yuanlong Fan and Yanhua Hong are with School of Computer Science and Electronic Engineering, Bangor University, Wales, LL57 1UT, United Kingdom (e-mail: y.fan@bangor.ac.uk; y.hong@bangor.ac.uk).

$\mathrm{Pu} \mathrm{Li}$ is with Key Laboratory of Advanced Transducers \& Intelligent Control System, Ministry of Education, Taiyuan University of Technology, and Institute of Optoelectronic Engineering, College of Physics \& Optoelectronics, Taiyuan University of Technology, Taiyuan, 030024, China (e-mail: lipu8603@126.com). spontaneous emission can significantly reduce or even eliminate the threshold of SNLs [3, 4]. Moreover, the opportunities for achieving high modulation bandwidth using SNLs have been suggested [5].

For most applications it would be expected that SNLs can operate stably. However, as the complexity of PICs increases with the incorporation of a variety of optical components, such stable operation may be compromised due to unwanted external optical feedback (EOF) into the SNL from such PIC components. Due to such EOF, SNLs may exhibit a variety of dynamical behaviours, e.g., periodic oscillations, quasi-periodic oscillations, and optical chaos [6,7]. As is well known, EOF effects can occur with only a small portion of light re-entering the SNL, and such effects cannot be prevented without convenient means for achieving optical isolation of order $-20 \mathrm{~dB}[8]$. There are three main controllable parameters that influence the stability of SNLs when subject to EOF. They are, respectively, the laser injection current $\left(I_{\mathrm{dc}}\right)$, the feedback strength $(\kappa)$, and the external cavity length $(L)$ (or the feedback phase $\phi_{0}$ ). It is thus of importance to establish the range of these parameters over which SNLs can remain stable. Note that external cavity length can also be expressed in a way that $L=L_{0}+\Delta L$, where $L_{0}$ is the initial external cavity length, and $\Delta L$ is a small variation of external cavity length within the range of $\left[0, \lambda_{0} / 2\right]$. Feedback phase $\phi_{0}$ is related to $L$ via $\phi_{0}=4 \pi L / \lambda_{0}=4 \pi L_{0} / \lambda_{0}+4 \pi \Delta L / \lambda_{0}$, where $\lambda_{0}$ is the wavelength of the solitary SNL.

Investigations of the stability of SNLs subject to optical feedback have been reported in [7]. However, [7] only considers cases of optical feedback where the feedback phase is fixed. In fact, the influence of feedback phase is of particular importance due to its laser-wavelength-scale sensitivity. For instance, a half-wavelength change of $L$ will lead to a phase change of $2 \pi$. Within such a change of phase, it has been seen in conventional semiconductor lasers that transitions between stable and unstable states can easily occur [9-16]. However, in practice, it is challenging to maintain a fixed $\phi_{0}$ even with use of precise phase control elements [14]. For SNLs which may be operated in photonic integrated circuits, it can be anticipated that the feedback phase will be difficult to control. Therefore, from a practical point of view, it is important to fully understand the effects of feedback phase in order that the SNL can be operated stably independently of the feedback phase.

In this paper, with a view to achieving a feedback-phase-independent stable SNL, we, first of all, investigate the influence of feedback phase with the aid of rate 
equations where the Purcell effect is included. This work reveals the impact of the feedback phase on the stability of SNLs. Attention is then given to the SNL stability with respect to feedback phase and feedback strength leading to the definition of a feedback-phase-independent stable region. Finally, consideration is given to aspects of stability associated with the injection current and initial external cavity length. This work provides practical guidelines for the design of a phase-independent stable SNL.

\section{MODEL}

The model used to describe the dynamics of semiconductor nanolasers (SNLs) in the presence of external optical feedback (EOF) is based on a modified form of Lang and Kobayashi rate equations [17], where enhanced spontaneous emission is incorporated via two factors: the Purcell factor, $F$ and an enhanced spontaneous emission coupling factor, $\beta$ (see (1)-(4)) as introduced in [18]. Note that this model can be used for Fabry-Perot (FP) lasers, but not for ring lasers.

$$
\begin{aligned}
\frac{d S(t)}{d t}= & \Gamma\left\{\frac{F \beta N(t)}{\tau_{n}}+\left[\frac{g_{n}\left(N(t)-N_{0}\right)}{1+\varepsilon S(t)}-\frac{1}{\Gamma \tau_{p}}\right] S(t)\right\} \\
& +2 \kappa \sqrt{S(t) S(t-\tau)} \cos (\theta(t))+F_{S}(t) \\
\frac{d \phi(t)}{d t}= & \frac{\alpha}{2} \Gamma g_{n}\left[N(t)-N_{0}-\frac{1}{g_{n} \tau_{p}}\right] \\
& -\kappa \frac{\sqrt{S(t-\tau)}}{\sqrt{S(t)}} \sin (\theta(t))+F_{\phi}(t) \\
\frac{d N(t)}{d t}= & \frac{I_{d c}}{e V_{a}}-\frac{N(t)}{\tau_{n}}[F \beta+(1-\beta)] \\
& -\frac{g_{n}\left[N(t)-N_{0}\right]}{1+\varepsilon S(t)} S(t)+F_{N}(t) \\
\theta(t)= & \omega_{0} \tau+\phi(t)-\phi(t-\tau)
\end{aligned}
$$

These two factors act together and so for computational purposes may be incorporated into one parameter. However, it is argued here that two separate physical effects are at work and hence their impacts can, in principle, be amended independently. One observes that SNLs are not necessarily single mode lasers and hence, for a given cavity, if the number of lasing modes excited changed say with laser drive current so would $\beta$. It is underlined that the Purcell factor and the spontaneous emission coupling factor impact the spontaneous emission rate as shown in (1) and (3) above. Specifically, it is pointed out that for Purcell factors greater than unity an effective reduction in the carrier lifetime will result. Similarly, an increase of the spontaneous emission coupling factor towards unity also causes an effective reduction of the carrier lifetime. In contrast, the phase in (2) is dependent on the laser gain and is thus not affected by the enhanced spontaneous emission. Enhanced spontaneous emission may be expected to increase the laser linewidth and thereby raising issues of the coherence length of SNLs. We are unaware of any publications detailing calculations of the linewidth of the SNL but published experimental results suggest that narrow linewidths occur in SNLs [19-21]. For example, in [19], with a centre wavelength of $1560.8 \mathrm{~nm}$, the linewidth of the nanolaser is $1.17 \mathrm{pm}(143.5$ $\mathrm{MHz}$ ) which gives a coherence length of $66.5 \mathrm{~cm}$.

In (1)-(4), $t$ is the time, $S(t)$ is the photon density, $N(t)$ is the carrier density and $\phi(t)$ is the phase, $\theta(t)$ is the phase change. The three main controllable parameters for an SNL are, respectively, the injection current $I_{\mathrm{dc}}$, the feedback strength $\kappa$, and the external cavity length $L$ (or feedback phase $\phi_{0}$ ). $\kappa=\eta\left(1-r_{2}\right)\left(r_{1} / r_{2}\right) c /\left(2 n L_{\text {in }}\right)$, where $\eta$ is the fraction of the reflected field which couples back into the lasing mode. $\eta=\left(f_{\text {ext }} r_{2}\right)^{1 / 2} / r_{1}$, where $f_{\text {ext }}$ is the feedback fraction (ratio of externally reflected light power versus emitted light power) [22]. $r_{1}=0.95$ and $r_{2}=0.85$ are the power reflectivities of the external object and the front laser facet respectively, $c$ is the speed of light, $n=3.4$ is the refractive index and $L_{\mathrm{in}}=1.39 \mu \mathrm{m}$ is the laser internal cavity length. Note that the external cavity roundtrip delay $\tau$ in (1), (2), and (4) is related to $L$ via $\tau=2 L / c$, where $L$ is the effective external cavity length. In a PIC, $L=n^{\prime} L^{\prime}$ where $n^{\prime}$ and $L^{\prime}$ are, respectively, the refractive index of the waveguide and the physical distance to the external reflector. $F_{S}(t), F_{\phi}(t)$ and $F_{N}(t)$ are Langevin noise sources used to model spontaneous emission noise [23]. They are calculated by:

$$
\begin{aligned}
& F_{S}(t)=\sqrt{\frac{2 \beta S(t) N(t)}{\tau_{n} \Delta T}} x_{S} \\
& F_{\phi}(t)=\frac{1}{S(t)} \sqrt{\frac{\beta S(t) N(t)}{\tau_{n} \Delta T}} x_{\phi} \\
& F_{N}(t)=-\sqrt{\frac{2 \beta S(t) N(t)}{\tau_{n} \Delta T}} x_{S}+\sqrt{\frac{2 N(t)}{\tau_{n} \Delta T V_{a}}} x_{N}
\end{aligned}
$$

where $x_{S}, x_{\phi}$ and $x_{N}$ are independent Gaussian distributed random variables with zero mean and unity variance for ensembles of time. $\Delta T$ is called Langevin noise application interval [23] which is the time slot of Langevin noise. The noise forces should describe a white noise spectrum at least up to the relaxation oscillation frequency [24] which is $6.3 \mathrm{GHz}$ for the SNL (as shown in Fig. 3(f) in Section IV). In this work $\Delta T$ is chosen to be $125 \mathrm{ps}$. This results in a power spectral density for the noise having its first zero at a frequency of $8 \mathrm{GHz}\left(\Delta \mathrm{T}^{-1}\right)$ which covers the relaxation oscillation frequency of the SNL, hence satisfying the condition that the noise spectrum is white up to a frequency greater than the relaxation oscillation frequency. The physical meanings and values of other symbols in (1) - (4) are listed in Table I.

A fourth order Runge-Kutta integration method was used to numerically solve (1)-(4). In the simulations, a temporal resolution of $\Delta t=1 \mathrm{ps}$ is selected and the duration of the time series is set to be $1 \mu s$ which leads to 1 million integration steps. The Gaussian distributed random variables $x_{S}, x_{\phi}$ and $x_{N}$ are calculated at the start of each integration step. The dynamics of SNLs, including stable and unstable states, can be determined from the temporal waveforms of the photon density $S(t)$ after the cessation of transient effects. Details for distinguishing 
TABLE I

PHYSICAL MEANING AND VALUE OF SYMBOLS IN (1)-(4)

\begin{tabular}{cll}
\hline \hline Symbol & Physical Meaning & Value \\
\hline$F$ & Purcell factor & variable \\
$I_{d c}$ & injection current & variable \\
$\kappa$ & feedback strength & variable \\
$\tau$ & external cavity roundtrip delay & variable \\
$\Gamma$ & confinement factor & 0.65 \\
$\beta$ & spontaneous emission coupling factor & 0.05 \\
$\tau_{n}$ & carrier life time & $1.00 \times 10^{-9} \mathrm{~s}$ \\
$g_{n}$ & differential gain & $1.65 \times 10^{-12} \mathrm{~m}^{3} / \mathrm{s}$ \\
$N_{0}$ & carrier density at transparency & $1.10 \times 10^{24} \mathrm{~m}^{-3}$ \\
$\varepsilon$ & gain saturation factor & $2.30 \times 10^{-23} \mathrm{~m}^{3}$ \\
$\tau_{p}$ & photon life time & $0.36 \times 10^{-12} \mathrm{~s}$ \\
$\alpha$ & line-width enhancement factor & 5 \\
$e$ & elementary charge & $1.60 \times 10^{-19} \mathrm{C}$ \\
$V_{a}$ & volume of the active region & $3.96 \times 10^{-19} \mathrm{~m}^{3}$ \\
$\omega_{0}$ & optical frequency & $1.18 \times 10^{15} \mathrm{rad} / \mathrm{s}$ \\
\hline \hline
\end{tabular}

different dynamics of SNLs are described in the following sections.

\section{INFLUENCE OF FEEDBACK PHASE ON SNL STABILITY}

In this section, the influence of feedback phase on the stability of SNLs is investigated using bifurcation diagrams which are informative and effective means widely used for investigating the dynamics of a system as a function of one of the system parameters [25-27].

Figure 1 (a)-(c) show the bifurcation diagrams of the SNLs as a function of feedback phase, $\phi_{0}$, with the feedback coupling fraction of $\eta=2.5 \times 10^{-3}, \quad \eta=4.5 \times 10^{-3}, \quad$ and $\eta=6.0 \times 10^{-3}$ respectively. In order to clearly identify the dynamic regimes, the bifurcation diagrams, as is usual, are obtained without considering noise. The regimes of different dynamics are distinguished by dashed lines in Fig. 1(a)-(c). These bifurcation diagrams are obtained by recording the local extremum of the time series of the photon density at every different point of $\phi_{0}$ which takes 300 equally spaced points within the range of $\phi_{0} \in$ [0,2 $\pi]$, corresponding to $L \in\left[L_{0}, L_{0}+\lambda_{0} / 2\right]$. For each point of $\phi_{0}$, the same initial conditions are employed, and the time series is also obtained to visually verify the dynamics of SNL. Note that the dynamics of a laser is $2 \pi$ periodic with respect to $\phi_{0}$ [28]. When the SNL is stable (S), only one extremum, can be found in the temporal waveform of photon density. When the SNL is unstable, two or more extrema are located, and the number of the extrema can be used to define different types of dynamics. For example, a few extrema indicate periodic oscillations (PO), clusters of extrema indicate quasi-periodic (QP) oscillations whereas many extrema indicate chaos (C). As shown in the following section the transitions between these dynamical behaviours can be identified both in the time domain (time series) and the frequency domain (power spectrum). It is noted that the simulations reveal no multi-state intermittency [29] which have been found in conventional lasers with EOF.

In Fig. 1 (a), where $\eta=2.5 \times 10^{-3}\left(f_{\text {ext }}=6.6 \times 10^{-6}\right)$, the SNL is stable only when $\phi_{0}$ is within the range $[0.47 \pi, 1.23 \pi]$. An
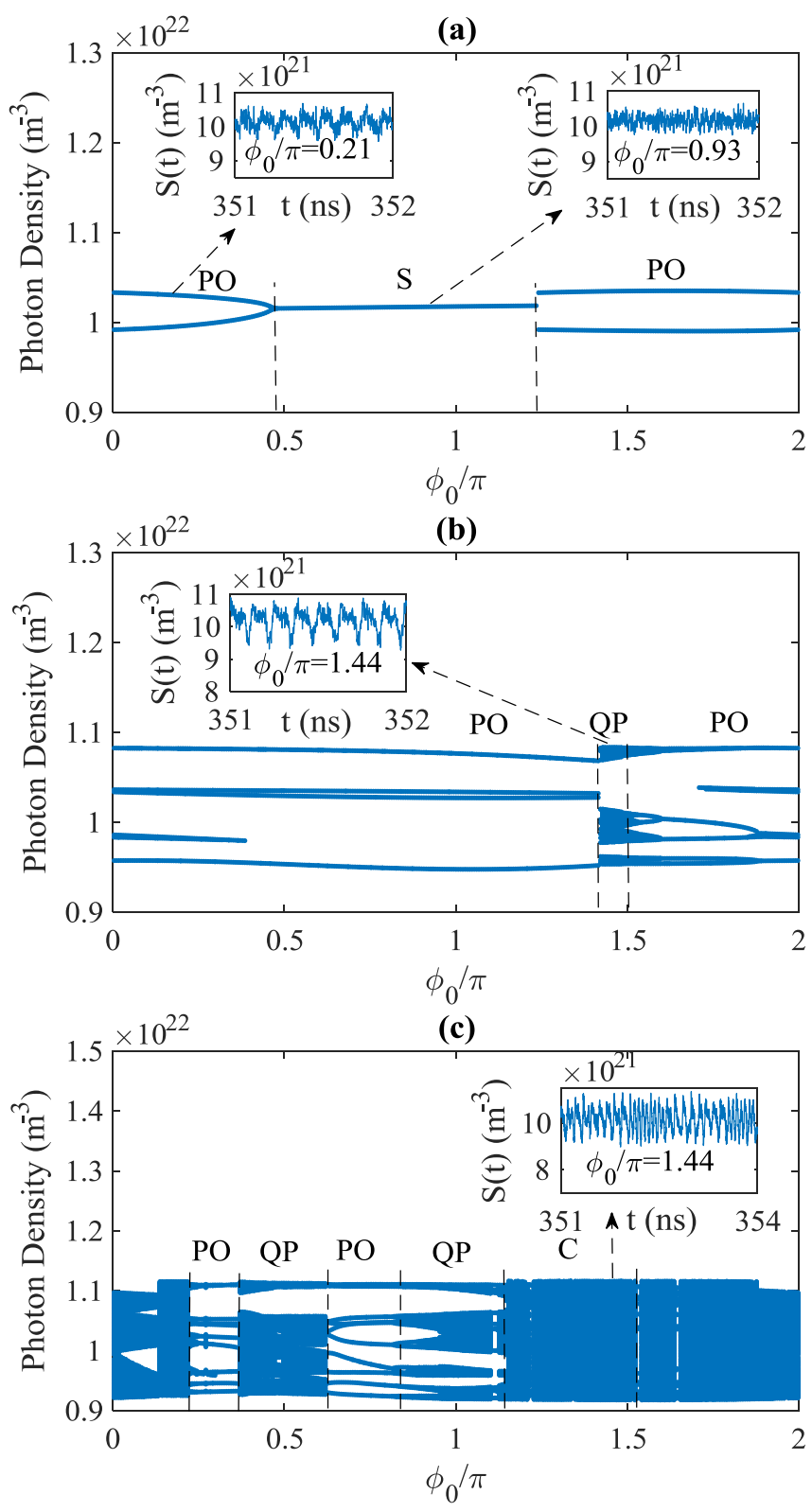

Fig. 1. Bifurcation diagrams in terms of feedback phase $\phi_{0}$ for a semiconductor nanolaser $(F=20, \beta=0.05)$ in presence of optical feedback where injection current $I_{\mathrm{dc}}=2.7 I_{\mathrm{th}}$ and initial external cavity length $L_{0}=15 \mathrm{~mm}\left(I_{\mathrm{th}}=1.1 \mathrm{~mA}\right.$ [7] is the threshold current). Dashed lines separate different dynamical regimes which include Stable (S), Periodic Oscillations (PO), Quasi-Periodic (QP) oscillations and Chaos (C). (a) $\eta=2.5 \times 10^{-3}\left(f_{\text {ext }}=6.6 \times 10^{-6}\right)$, (b) $\eta=4.5 \times 10^{-3}$ $\left(f_{\text {ext }}=2.2 \times 10^{-5}\right)$, (c) $\eta=6.0 \times 10^{-3}\left(f_{\text {ext }}=3.8 \times 10^{-5}\right)$. Insets respectively represent temporal waveforms of $S(t)$ in stable operation, periodic oscillations, quasi-periodic oscillations and chaos for particular feedback phases.

example of a stable time series of $S(t)$ when $\phi_{0}=0.93 \pi$ is shown in the inset of Fig. 1(a). Note that this example and the other time series insets of Fig. 1 are obtained taking account of Langevin noise. When $\phi_{0}=0.47 \pi$, the relaxation oscillation of the SNL becomes self-sustained leading to a periodic oscillation of the photon density. An example of such periodic oscillations is shown in the inset of Fig. 1 (a) where $\phi_{0}=0.21 \pi$.

With an increase of feedback coupling factor to $\eta=4.5 \times 10^{-3}$ $\left(f_{\text {ext }}=2.2 \times 10^{-5}\right)$, the SNL is always unstable irrespective of the value of $\phi_{0}$, as shown in Fig. 1 (b). Moreover, the order of periodic oscillations increases with the increase of feedback 
and quasi-periodic oscillations start to appear. An example of quasi-periodic oscillations is shown in the inset of Fig. 1(b) where $\phi_{0}=1.44 \pi$.

When $\eta$ further increases to $6.0 \times 10^{-3} \quad\left(f_{\text {ext }}=3.8 \times 10^{-5}\right)$, as shown in Fig. 1 (c), the SNL becomes even more unstable as evidenced by the fact that chaos almost occupies half of the bifurcation diagram with the remainder of the diagram being occupied by higher order of periodic oscillations and quasi-periodic oscillations. An example of chaos is shown in the inset of Fig. 1 (c) where $\phi_{0}=1.44 \pi$.

Based on the bifurcation diagrams in Fig. 1, it is clear to see that the feedback phase can significantly influence the dynamics of the SNL. It should be also noted that precise control of feedback phase is almost impossible in photonic integrated circuits due to the laser-wavelength-scale sensitivity of the feedback phase. Therefore, it is highly desirable to identify a region where the stability of the SNL is independent of the feedback phase. In the next section, this issue is approached by investigating the stability map of the SNL where a phase independent stable region is indeed found.

\section{FeEdBack Phase IndePEndent Stable SNLS}

\section{A. Stability Map}

The stability map is constructed by numerically solving (1)-(4) with respect to the feedback coupling factor and phase which both take 150 points equally spaced within the ranges of $\eta \in\left[0.0,6.0 \times 10^{-3}\right]$ and $\phi_{0} \in[0,2 \pi]$ respectively. Due to the presence of the noise some care needs to be taken in defining the boundary between the stable and unstable states. To this end, we define a measure which characterizes that transition. Specifically, we define the parameter $\delta$ as follows:

$$
\delta=\frac{\sigma_{S}-\sigma_{0}}{\sigma_{0}}
$$

where $\sigma_{S}$ and $\sigma_{0}$ are respectively the standard deviation of the time series of the photon density for the SNL with and without EOF. When $\delta$ is larger than $10 \%$, the SNL is considered here to be unstable.

Figure 2(a) shows the stability map of an SNL ( $F=20, \beta=0.05)$ when $I_{\mathrm{dc}}=2.7 I_{\mathrm{th}}, L_{0}=15 \mathrm{~mm}$. The blue shaded region is the unstable region which is separated from the stable region by the stability limit (red in Fig. 2(a)). From Fig. 2, it is seen that below some value of the feedback coupling factor a feedback-phase-independent stable SNL is obtained. We define this minimum feedback coupling factor as critical feedback coupling factor and denote it as $\eta_{\mathrm{c}}$ (shown as dashed line in Fig. 2(a)). It is worth to mention that there is also a tilted stable region above the dashed line in Fig. 2(a). This region can be utilized for stable operation if the phase can be tuned over some range.

To further verify the stability map in Fig. 2(a), both the time series and the power spectra for five different values of $\eta$ when $\phi_{0}=1.45 \pi$ are presented in Fig. 3. These five $\eta$ values are, respectively, $\eta_{1}=1.0 \times 10^{-3}, \eta_{2}=1.6 \times 10^{-3}, \eta_{3}=1.9 \times 10^{-3}$, $\eta_{4}=4.0 \times 10^{-3}$ and $\eta_{5}=5.8 \times 10^{-3}$ which are shown as green dots in Fig. 2(a). An enlarged view of the circled area in Fig. 2(a)

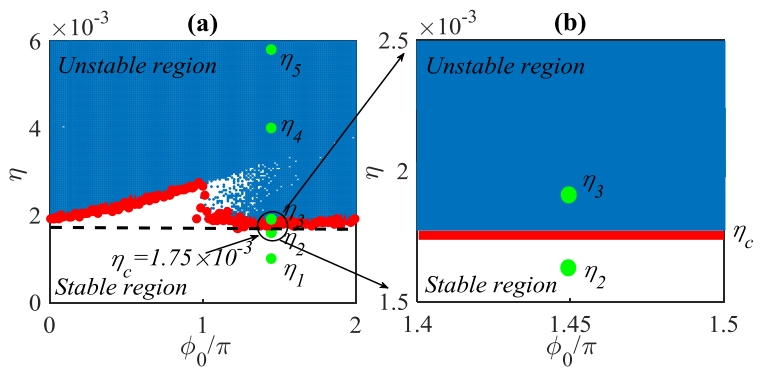

Fig. 2. The stability map of an SNL $(F=20, \beta=0.05)$ when $I_{\mathrm{dc}}=2.7 I_{\text {th }}$ and $L_{0}=15$ $\mathrm{mm}$. (a) The stability map where the blue shaded region is the unstable region which is separated from the stable region by the stability limit (shown as the red line). (b) An enlarged view of the circled area in Fig. 2(a). $\eta_{1}-\eta_{5}$ : Feedback strength used to verify the stability map. $\eta_{\mathrm{c}}$ : the minimum value of $\eta$ on the stability limit below which a feedback-phase-independent stable SNL is guaranteed.
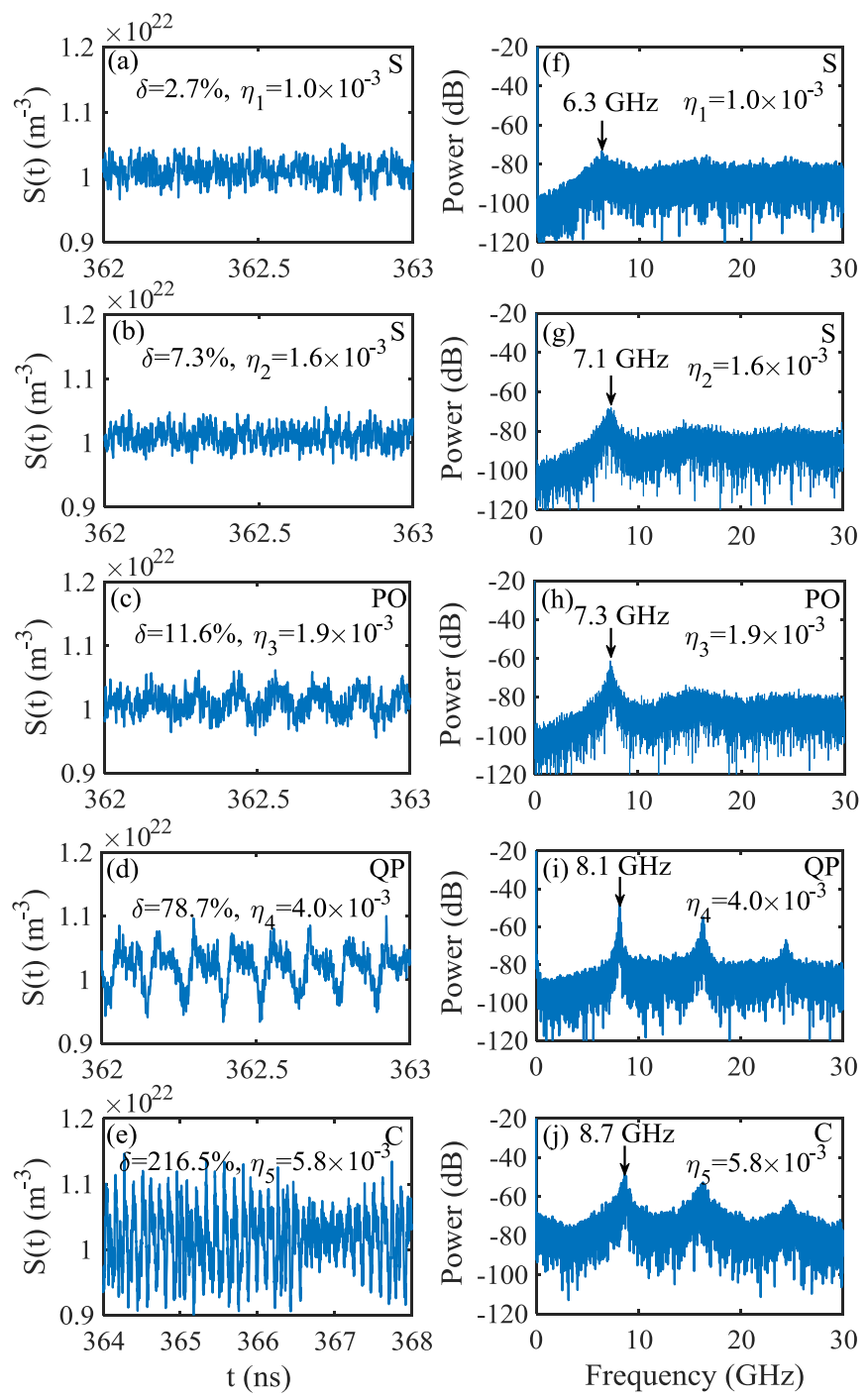

Fig. 3. Time series and power spectra for different values of $\eta$ shown in Fig. 2(a). (a)-(e) Time series. (f)-(j) Power spectra.

shows that $\eta_{2}$ and $\eta_{3}$, respectively, are slightly below and above the stability boundary (as shown in Fig. 2(b)). From Fig. 3, it can be seen that when $\eta_{1}=1.0 \times 10^{-3}$, the SNL is stable (S) where $\delta=2.7 \%$. This stability can also be confirmed by the power spectrum (Fig. 3(f)) where there are small and broad components at around $6.3 \mathrm{GHz}$ that corresponds to the 
relaxation oscillation frequency of the solitary SNL. The fluctuations in the time series of the photon density (Fig. 3(a)) are, of course, due to the Langevin noises.

With increase of feedback coupling factor to $\eta_{2}=1.6 \times 10^{-3}$ which is slightly below the stability boundary (as shown in Fig. 2(b)), the SNL remains stable $(\delta=7.3 \%)$ as shown in Fig. 3(b) and a peak appears in the spectrum at $7.1 \mathrm{GHz}$ (Fig. 3(g)) showing the SNL is about to enter the unstable region.

When the SNL enters the unstable region where $\eta_{3}=1.9 \times 10^{-3}$, the photon density undergoes periodic oscillations (PO) with $\delta=11.6 \%$ (Fig. 3 (c)). The frequency of the peak in the spectrum becomes sharper and shifts from the stand-alone laser relaxation oscillation frequency to $7.3 \mathrm{GHz}$ (Fig. 3(h)) due to the EOF [30].

With further increase of the feedback coupling factor to $\eta_{4}=4.0 \times 10^{-3}$, the photon density exhibits quasi-periodic (QP) dynamics with $\delta=78.7 \%$ (Fig. 3(d)) as evidenced by the appearance of other peaks in the spectrum (Fig. 3(i)).

When the feedback coupling factor increases to a higher value, i.e., $\eta_{5}=5.8 \times 10^{-3}$, the peaks in the spectrum are broadened and the floor level increases (Fig. 3(j)) compared with the cases of S, PO and QP. These two phenomena indicate the occurrence of chaos (C) (Fig. 3(e)) [30]. In this case, $\delta=216.5 \%$.

Based on the results in Fig. 3, it can be seen that the SNL follows a quasi-periodic route to chaos as the feedback strength increases thereby confirming the significance of the stability boundary in Fig. 2.

After identifying $\eta_{\mathrm{c}}$, it is natural to consider how $\eta_{\mathrm{c}}$ depends on the other two main system parameters, i.e., the injection current, $I_{\mathrm{dc}}$, and initial external cavity length, $L_{0}$, and hence to explore the opportunities for achieving stable operations of the SNL over a wider operating range. To achieve this, the following two sets of simulations are performed.

\section{B. Relationship Between $\eta_{c}$ and $I_{d c}$}

The first set of simulations investigates the relationship between $\eta_{\mathrm{c}}$ and $I_{\mathrm{dc}}$ for three different SNLs all with a fixed initial external cavity length $L_{0}=15 \mathrm{~mm}$. In the simulation, we set the $I_{\mathrm{dc}}$ within the range of $I_{\mathrm{dc}} \in\left[1.4 I_{\mathrm{th}}, 4.1 I_{\mathrm{th}}\right]$ with 25 equally spaced points. For each point, we numerically generate a stability map and record the corresponding $\eta_{\mathrm{c}}$. The relationships between $\eta_{\mathrm{c}}$ and $I_{\mathrm{dc}}$ of three different SNLs are shown in Fig. 4 where circles, diamonds and squares respectively represent SNLs with $\beta=0.05$ and Purcell factors $F=20,40$ and 60. Furthermore, the relationships between $\eta_{\mathrm{c}}$ and $I_{\text {dc }}$ can be described by (9) obtained by using curve fitting technique [31], where $A_{1}, A_{2}, A_{3}$ and $A_{4}$ are functions of $F$. The values of $A_{1}, A_{2}, A_{3}$ and $A_{4}$ are listed in Table II. The fitting results are plotted in Fig. 4, where green, yellow and red solid lines respectively represent for $F=20,40$ and 60 .

$$
\eta_{c}\left(I_{d c}, F\right)=A_{1}(F) e^{A_{2}(F) I_{d c}}+A_{3}(F) e^{A_{4}(F) I_{d c}}
$$

From Fig. 4, we can see that the results obtained from (9) match well the numerical results where $\eta_{\mathrm{c}}$ increases bi-exponentially with the decrease of injection current. Such a trend indicates that a low injection current is desired to achieve a feedback-phase-independent stable SNL that is able to resist high feedback strength. Clearly for lower injection currents the laser emission is lower and hence the reflected light intensity is lower, thus leading to a more stable SNL. However, a low injection current also leads to a low modulation bandwidth [32]. Therefore, there is a tradeoff between the stability and modulation bandwidth of SNL. Careful choice should be made of the injection current when a feedback-phase- independent stable SNL is sought.

Further, in Fig. 4, it can be seen $\eta_{\mathrm{c}}$ increases with increase of Purcell factor and the increment of $\eta_{\mathrm{c}}$ decreases with the increase of injection current. The enhancement of Purcell factor can be achieved in an SNL by enhancing the $Q$ factor [33]. This is possible with changes in the nanolaser design by using highly reflective mirrors which can be attained by the use of metal coat [34-40], or by adjusting claddings on top of the active region to eliminate mode coupling [41, 42].

TABLE II

VALUES OF $A_{1}, A_{2}, A_{3}$ AND $A_{4}$ FOR DIFFERENT $F$

\begin{tabular}{ccccc}
\hline \hline$F$ & $A_{1}$ & $A_{2}$ & $A_{3}$ & $A_{4}$ \\
\hline 20 & 0.010 & -2700 & 0.0017 & -4.36 \\
40 & 0.009 & -1420 & 0.0026 & -28.39 \\
60 & 0.082 & -2338 & 0.0038 & -84.18 \\
\hline \hline
\end{tabular}

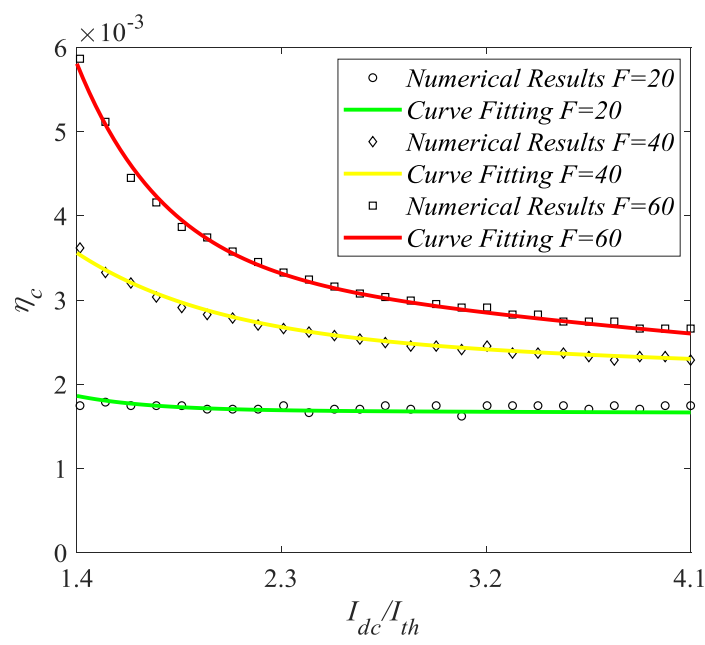

Fig. 4. The relationship between $\eta_{\mathrm{c}}$ and $I_{\mathrm{dc}}$ of three different SNLs with $\beta=0.05$ and Purcell factors are respectively $F=20,40$ and 60 . The initial external cavity length $L_{0}=15 \mathrm{~mm}$. Circles, diamonds and squares are numerical results respectively when $F=20,40$ and 60 . Green, yellow and red solid lines are results respectively obtained from (9) for $F=20,40$ and 60.

\section{Relationship between $\eta_{c}$ and $L_{0}$}

The second set of simulations investigates the relationship between $\eta_{\mathrm{c}}$ and $L_{0}$ for three different SNLs all with a fixed injection current $I_{\mathrm{dc}}=2.7 I_{\mathrm{th}} . L_{0}$ is set within the range of $L_{0} \in[0$ $\mathrm{mm}, 50 \mathrm{~mm}$ ] also with 25 equally spaced points. Note that such a range is much less than the coherence length calculated using the linewidth in [19], i.e., $66.5 \mathrm{~cm}$. The simulation results are plotted in Fig. 5 where circles, diamonds and squares still respectively represent SNLs with $\beta=0.05$ and different Purcell factors $F=20,40$ and 60 .

In Fig. 5, we also plot the relationship between $\eta_{\mathrm{c}}$ and $L_{0}$ for the three SNLs using the following bi-exponential expression: 


$$
\eta_{c}\left(L_{0}, F\right)=B_{1}(F) e^{B_{2}(F) L_{0}}+B_{3}(F) e^{B_{4}(F) L_{0}}
$$

where $B_{1}, B_{2}, B_{3}$ and $B_{4}$ are functions of $F$. Table III lists the values of $B_{1}, B_{2}, B_{3}$ and $B_{4}$ for $F=1,20,40$ and 60 .

TABLE III

VALUES OF $B_{1}, B_{2}, B_{3}$, AND $B_{4}$ FOR DIFFERENT $F$

\begin{tabular}{ccccc}
\hline \hline$F$ & $B_{1}$ & $B_{2}$ & $B_{3}$ & $B_{4}$ \\
\hline 1 & 0.009 & -251.0 & 0.0019 & -10.850 \\
20 & 0.009 & -265.2 & 0.0016 & -0.243 \\
40 & 0.006 & -161.9 & 0.0019 & 3.997 \\
60 & 0.006 & -138.1 & 0.0021 & 6.087 \\
\hline \hline
\end{tabular}

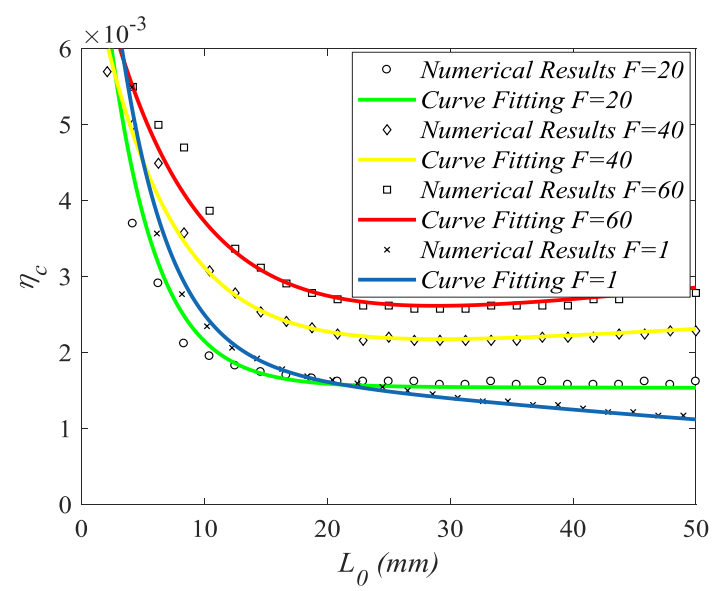

Fig. 5. The relationship between $\eta_{\mathrm{c}}$ and $L_{0}$ of three different SNLs with $\beta=0.05$ and Purcell factors $F=20,40$ and 60 . The injection current $I_{\mathrm{dc}}=2.7 I_{\mathrm{th}}$. Circles, diamonds and squares are numerical results for $F=20,40$ and 60 . Green, yellow and red solid lines are results respectively obtained from (10) for $F=20,40$ and 60. Crosses are numerical results for a conventional laser where $F=1$ and $\beta=1 \times 10^{-5}$. The blue solid line is obtained from (10) for $F=1$.

From Fig. 5, it can be seen that, with the decrease of initial external cavity length, $\eta_{\mathrm{c}}$ firstly decreases and then slightly increases both in a bi-exponential way as described by (10) for a relatively large $F$, e.g., $F=40$ and 60 . For $F=20, \eta_{\mathrm{c}}$ increases bi-exponentially with the decrease of $L_{0}$. This confirms that sources of reflection should be placed as close as possible to the SNL in order to ensure a stable SNL. Note that for a relatively short external cavity length (e.g., $L_{0}<4 \mathrm{~mm}$ ), the SNLs are always feedback-phase-independent stable even under strong feedback. It is noteworthy that such behaviour is in line with that found in conventional lasers with $L_{0}=4.5 \mathrm{~mm}$ as reported in [26, 43]. In Fig. 5, we plot the relationship between $\eta_{\mathrm{c}}$ and $L_{0}$ for $F=1$ and $\beta=1 \times 10^{-5}$ which are typical values for conventional lasers. The crosses and blue solid line in Fig. 5 are respectively the numerical and curve fitting results for the conventional laser. In contrast, results in [13, 44, 45] showed that the feedback phase can destabilize the laser for short external cavities and certain feedback strengths. We point that in the present work and in [26, 43], effects of nonlinear gain have been taken into account whereas such effects were not considered in [13, 44, 45].

\section{CONCLUSION}

In this paper, the stability of semiconductor nanolasers (SNLs) with external optical feedback (EOF) is investigated in order to achieve a feedback-phase-independent stable SNL. Based on the investigations, a critical feedback coupling fraction $\eta_{\mathrm{c}}$ is identified, below which the SNL operates stably independent of the feedback phase. Further, this critical $\eta_{\mathrm{c}}$ is found to decrease with increasing injection current and initial external cavity length, and to increase with increasing Purcell factor. The results presented in this paper provide practical guidelines for assessing the stability of SNLs within the densely integrated photonic circuits where the feedback phase is inevitable and difficult to control, which is important for designing phase-independent stable SNLs for various photonic integrated circuit (PIC) based applications such as optical communications and sensing.

\section{ACKNOWLEDGMENT}

Authors appreciate Prof. K. Alan Shore's useful discussions and constructive suggestions.

\section{REFERENCES}

[1] Q. Gu and Y. Fainman, Semiconductor Nanolasers. Cambridge, U.K.: Cambridge University Press, 2017.

[2] E. M. Purcell, "Spontaneous emission probabilities at radio frequencies," Phys. Rev., vol. 69, pp. 681, 1946.

[3] M. Khajavikhan et al., "Thresholdless nanoscale coaxial lasers," Nature, vol. 482, pp. 204-207, Feb. 2012.

[4] Y. Ota et al., "Thresholdless quantum dot nanolaser," Opt. Express, vol. 25, no. 17, pp. 19981-19994, Aug. 2017.

[5] K. A. Shore, "Modulation bandwidth of metal-clad semiconductor nanolasers with cavity-enhanced spontaneous emission," Electron. Lett., vol. 46, no. 25, pp. 1688 - 1689, Dec. 2010.

[6] D. M. Kane and K. A. Shore, Unlocking Dynamical Diversity: Optical Feedback Effects on Semiconductor Lasers. Chichester, U.K.: John Wiley \& Sons Ltd, 2005.

[7] Z. A. Sattar and K. A. Shore, "External optical feedback effects in semiconductor nanolasers," IEEE J. Sel. Topics Quantum Electron., vol. 21, no. 6, pp. 1800106, Nov./Dec. 2015.

[8] M. J. R. Heck, "Grating coupler enabled optical isolators and circulators for photonic integrated circuits," IEEE J. Sel. Topics Quantum Electron., vol. 21, no. 4, pp. 6100109, Jul./Aug. 2015.

[9] R. W. Tkach and A. R. Chraplyvy, "Regimes of feedback effects in $1.5 \mu \mathrm{m}$ distributed feedback lasers," J. Lightw. Technol., vol. 4, no. 11, pp. 1655-1661, Nov. 1986.

[10] M. Wolfrum and D. Turaev, "Instabilities of lasers with moderately delayed optical feedback," Opt. Commun., vol. 212, no. 1-3, pp. 127-138, Oct. 2002.

[11] S. Wieczorek, B. Krauskopf, T. Simpson, and D. Lenstra, "The dynamical complexity of optically injected semiconductor lasers." Phy. Rep., vol. 416, no. 1, pp. 1-128, Sep. 2005.

[12] S. Behnia, K. Mabhouti, A. Jafari, and A. Akhshani, "Observations on the dynamics of external cavity semiconductor lasers," Optik - International J. Light and Electron Opt., vol. 123, no. 17, pp. 1555-1561, Sep. 2012.

[13] S. Donati and M. T. Fathi, "Transition from short-to-long cavity and from self-mixing to chaos in a delayed optical feedback laser," IEEE $J$. Quantum Electron., vol. 48, no. 10, pp. 1352-1359, Oct. 2012.

[14] D. Lenstra, "Relaxation oscillation dynamics in semiconductor diode lasers with optical feedback," IEEE Photon. Technol. Lett., vol. 25, no. 6, pp. 591-593, Mar. 2013.

[15] D. D'Agostino, H. P. M. M. Ambrosius, M. K. Smit, and D. Lenstra, "Integrated laser with optical feedback shows suppressed relaxation-oscillation dynamics." IEEE Photon. Technol. Lett. Vol. 27, no. 21, pp. 2292-2295, Nov. 2015.

[16] D. Zhao, S. Andreou, W. Yao, D. Lenstra, K. Williams and X. Leijtens, "Monolithically integrated multiwavelength laser with optical feedback: damped relaxation oscillation dynamics and narrowed linewidth," IEEE Photon. J., vol. 10. No. 6, Dec. 2018.

[17] R. Lang and K. Kobayashi, "External optical feedback effects on semiconductor injection laser properties," IEEE J. Quantum Electron., vol. 16, no. 3, pp. 347-355, Mar. 1980. 
[18] T. Suhr, N. Gregersen, K. Yvind, and J. Mørk, "Modulation response of nanoLEDs and nanolasers exploiting Purcell enhanced spontaneous emission," Opt. Express, vol. 18, no. 11, pp. 11230-11241, May 2010.

[19] J. Kim, et al., "Narrow linewidth operation of buried-heterostructure photonic crystal nanolaser," Opt. Express, vol. 20, no. 11, pp. 11643-11651, May 2012

[20] A. Bazin, et al., "Thermal management in hybrid InP/silicon photonic crystal nanobeam laser," Opt. Express, vol. 22, no. 9, pp. 10570-10578, May 2014.

[21] G. Crosnier et al., "Hybrid indium phosphide-on-silicon nanolaser diode," Nat. Photon., vol. 11, pp. 297-300, Apr. 2017.

[22] N. Schunk and K. Petermann, "Numerical analysis of the feedback regimes for a single-mode semiconductor laser with external feedback," IEEE J. Quantum Electron., vol. 24, no. 7, pp. 1242-1247, Jul. 1988.

[23] L. N. Langley and K. A. Shore, "Intensity noise and linewidth characteristics of laser diodes with phase conjugate optical feedback," IEE Proc. - Optoelectronics, vol. 141, no. 2, pp. 103-108, Apr. 1994.

[24] N. Schunk and K. Petermann, "Noise analysis of injection-locked semiconductor injection lasers," IEEE J. Quantum Electron., vol. 22, no. 5, pp. 642-650, May 1986.

[25] J. Awrejcewicz, Bifurcation and Chaos in Simple Dynamical Systems. Singapore: World Scientific, 1989.

[26] J. Mørk, B. Tromborg, and J. Mark, "Chaos in semiconductor lasers with optical feedback: theory and experiment," IEEE J. Quantum Electron., vol. 28, no. 1, pp. 93-108, Jan. 1992

[27] N. Li, H. Susanto, B. R. Cemlyn, I. D. Henning, and M. J. Adams, "Stability and bifurcation analysis of spin-polarized vertical-cavity surface-emitting lasers." Phys. Rev. A, vol. 96, no. 1, 013840, Jul. 2017.

[28] B. Haegeman, K. Engelborghs, D. Roose, D. Pieroux, and T. Erneux, "Stability and rupture of bifurcation bridges in semiconductor lasers subject to optical feedback," Phy. Rev. E, vol. 66, p. 046216, Oct. 2002.

[29] D. Choi, M. J. Wishon, C. Y. Chang, D. S. Citrin, and A. Locquet, "Multistate intermittency on the route to chaos of a semiconductor laser subjected to optical feedback from a long external cavity," Chaos, vol. 28 , no. 1, pp. 011102, Jan. 2018.

[30] A. Uchida, Optical Communication with Chaotic Lasers: Applications of Nonlinear Dynamics and Synchronization. Weinheim: Wiley-VCH, 2012.

[31] S. Arlinghaus, Practical Handbook of Curve Fitting. CRC Press, 1994.

[32] Z. A. Sattar and K. A. Shore, "Analysis of the direct modulation response of nanowire lasers," J. Lightw. Technol., vol. 33, no. 14, pp. 3028-3033, Jul. 2015.

[33] Q. Gu et al., "Purcell effect in sub-wavelength semiconductor lasers," Opt. Express, vol. 21, no. 13, pp. 15603-15617, Jul. 2013.

[34] M. T. Hill et al., "Lasing in metallic-coated nanocavities," Nat. Photon., vol. 1, pp. 589-594, Oct. 2007

[35] K. Ding, M. T. Hill, Z. C. Liu, L. J. Yin, P. J. van Veldhoven, and C. Z. Ning, "Record performance of electrical injection sub-wavelength metallic-cavity semiconductor lasers at room temperature," Opt. Express, vol. 21, no. 4, pp. 4728-4733, Feb. 2013.

[36] Q. Gu et al., "Amorphous $\mathrm{Al}_{2} \mathrm{O}_{3}$ shield for thermal management in electrically pumped metallo-dielectric nanolasers," IEEE J. Quantum Electron., vol. 50, no. 7, pp. 499-509, Jul. 2014.

[37] A. Mizrahi, V. Lomakin, B. A. Slutsky, M. P. Nezhad, L. Feng, and Y. Fainman, "Low threshold gain metal coated laser nanoresonators," Opt. Lett., vol. 33, no. 11, pp. 1261-1263, Jun. 2008.

[38] M. P. Nezhad et al., "Room-temperature subwavelength metallo-dielectric lasers," Nat. Photon., vol. 4, pp. 395-399, Jun. 2010.

[39] J. H. Lee et al., "Electrically pumped sub-wavelength metallo-dielectric pedestal pillar lasers," Opt. Express, vol. 19, no. 22, pp. 21524-21531, Oct. 2011.

[40] Q. F. Yao et al., "High-Q modes in defected microcircular resonator confined by metal layer for unidirectional emission," Opt. Express, vol. 21, no. 2, pp. 2165-2170, Jan. 2013.

[41] Y. D. Yang, Y. Z. Huang, W. H. Guo, Q. Lu, and J. F. Donegan, "Enhancement of quality factor for TE whispering-gallery modes in microcylinder resonators," Opt. Express, vol. 18, no. 12, pp. 13057-13062, Jun. 2010.

[42] X. M. Lv et al., "Analysis of vertical radiation loss and far-field pattern for microcylinder lasers with an output waveguide," Opt. Express, vol. 21, no. 13, pp. 16069-16074, Jul. 2013.

[43] N. Schunk and K. Petermann, "Stability analysis for laser diodes with short external cavities," IEEE Photon. Technol. Lett., vol. 1, no. 3, pp. 49-51, Mar. 1989.
[44] R. J. Jones, P. S. Spencer, J. Lawrence and D. M. Kane, "Influence of external cavity length on the coherence collapse regime in laser diodes subject to optical feedback," IEE Proc. - Optoelectronics, vol. 148, no. 1, pp. 7-12, Feb. 2001

[45] S. Donati and R. H. Horng, "The diagram of feedback regimes revisited," IEEE J. Sel. Topics Quantum Electron., vol. 19, no. 4, pp. 1500309, Jul./Aug. 2013.

Yuanlong Fan received the B. E. degree from Dalian University of Technology, China, in 2009 and his M.E. and Ph.D. degrees from the University of Wollongong, Australia, in 2011 and 2016, respectively. He was a recipient of the Australian Endeavour Fellowship to undertake postdoctoral research at the University of Wollongong, Australia in 2016.

$\mathrm{He}$ is currently a research officer at the School of Computer Science and Electronic Engineering, Bangor University, United Kingdom. His research interests include design of semiconductor nanolasers, nonlinear dynamics of semiconductor (nano)lasers, fiber nonlinear optics, and optical signal processing.

Yanhua Hong (M'15) received the B.Sc. degree in physics from Fujian Normal University, Fuzhou, China, in 1987, the M.S. degree in physics from Beijing Normal University, Beijing, China, in 1990, and the Ph.D. degree in optics from the Institute of Physics, Chinese Academy of Sciences, Beijing, in 1993.

She was a Lecturer with Beihang University, Beijing in 1993, and associate professor in 1995. Since 1997, she has been with Bangor University, Bangor, U.K., where she became a Permanent Research Staff in 2007, Lecturer in 2013, and Senior Lecturer in 2016. She is the author or coauthor of more than 180 journal and conference papers. Her current research interests include nonlinear dynamics in edge-emitting semiconductor lasers, vertical-cavity surface-emitting lasers, nano-lasers, semiconductor optical amplifiers, and dynamic networking for future optical networks.

Dr. Hong is a Senior Member of the Optical Society of America.

Pu Li received the M. S. degree in physical electronics from Taiyuan University of Technology (TYUT), Shanxi, China, in 2011. In 2014, he received the Ph. D. degree in circuits and systems at the Key Laboratory of Advanced Transducers and Intelligent Control System (Ministry of Education of China), College of Physics and Optoelectronics, TYUT, Shanxi, China.

In 2014, He joined TYUT. In 2017, He was a Visiting Scholar at the School of Electronic Engineering, Bangor University, United Kingdom. In 2018, he became a Professor at the College of Physics and Optoelectronics, TYUT. His research interests include nonlinear dynamics of semiconductor lasers and its applications, and all-optical signal processing.

Dr. Li is a Member of the Chinese Optical Society and the Chinese Physical Society. He also serves as a reviewer for journals of the OSA and Elsevier organizations. 